
REVIEWS NEUROSCIENCE

\title{
Exploring the mind field
}

A

t the turn of the twentieth century, the syndromes we now know of as Parkinson's disease, Alzheimer's disease and amyotrophic lateral sclerosis (ALS) were first beginning to acquire separate identities for neurologists. By the time the US Library of Congress and the National Institute of Mental Health declared the 1990s the 'Decade of the Brain', the distinctions were again beginning to blur. A key lesson reinforced since 1990 is that the processes that contribute to neurodegenerative disease are not exclusive to each disorder-rather, there are many common mechanisms. The last 15 years have been a true era of discovery for the brain.

To celebrate this progress, Nature Medicine and Nature Reviews Neuroscience present this collaborative series of review articles and perspectives written by leaders in the field. We have organized this supplement by focusing on cellular processes, rather than diseases, to highlight the many commonalities among seemingly distinct neurodegenerative disorders.

Our joint effort kicks off with a review by Ella Bossy-Wetzel, Robert Schwarzenbacher and Stuart Lipton that provides a general overview of shared molecular pathways in neurodegeneration. The supplement goes into further mechanistic detail with a review on protein aggregation and cellular toxicity by Christopher Ross and another on oxidative stress by Julie Andersen. Claudio Soto and Joaquin Castilla visit prion-related diseases and the prion hypothesis of neurodegeneration.

Miquel Vila and Serge Przedborski focus on one corner of a fast-expanding field: the genetic contributions to neurodegenerative disease. The discovery of mutations that cause early-onset Parkinson's disease, familial Alzheimer's disease, ALS and frontotemporal dementia not only makes possible genetic screening for affected individuals and their families, but also has pointed researchers towards essential molecular processes that underlie these disorders.

Despite a genetic component, age is the greatest risk factor in sporadic forms of neurodegenerative disease. Edward Koo and Raphael Kopan evaluate the possibility that altered presenilin activity may contribute to neurodegeneration in nongenetic or age-associated cases of neurodegenerative disease.

The therapeutic window of opportunity often comes during the presymptomatic initial stages of disease. Peter Nestor, Philip Scheltens and John Hodges examine the very early stages of Alzheimer's disease and review possible approaches to the presymptomatic identification of people who might benefit from early treatment. Despite our increased understanding of underlying pathogenesis, progress towards small-molecule therapies for neurodegenerative diseases has been modest. In his contribution, Peter Lansbury highlights the obstacles facing drug discovery and suggests new approaches for overcoming them.

Research over the past 15 years has overturned the dogma that we are born with all the neurons we will ever have. We now know that a population of brain stem cells is constantly dividing. The review by Zaal Kokaia, Alberto Martinez-Serrano and Olle Lindvall explores the promise and reality of using endogenous and exogenous stem cells for cell replacement as a therapeutic strategy for neurodegenerative disease.

The accompanying website (http://www.nature.com/ focus/neurodegen/) provides free access to this content for three months. We also acknowledge the generous financial support of AstraZeneca. Nature Medicine and Nature Reviews Neuroscience retain sole responsibility for editorial content and peer review. We hope you will find this supplement informative and stimulating. 